\title{
Construction of Double Cladding Small Dispersion Photonic Crystal Fiber to Guide Ultrashort Pulse at $800 \mathrm{~nm}$
}

\author{
H. StaWska* AND E. Bereś-PAWLiK \\ Radiocommunication and Teleinformatics Department, Wrocław University of Technology \\ Wybrzeże Wyspiańskiego 27, 50-370 Wrocław, Poland
}

The methods using femtosecond lasers are very popular in the world recently. They are used for different research methods. We begin to use a multi-photon autofluorescence method for cancer tissue detection. We plan to use this method with special photonic crystal fiber optic sensors. We would like to use to this detection femtosecond oscillator for $800 \mathrm{~nm}$ region. However, according to our information there is no fiber which can provide these femtosecond signals at $800 \mathrm{~nm}$ region without dispersion and nonlinear effect. Applying typical fiber causes dispersion of guided signals. Therefore we are preparing suitably designed fibers. Double-clad photonic fibers can play a dual role of ultrashort pulse delivery and efficient collection of nonlinear optical signals. In the paper a new design of double cladding small dispersion photonic crystal fiber is proposed. Simulations of both structure and dispersion in proposed fibers are presented.

PACS: 42.81.-i, 42.81.Pa, 42.62.Be, 42.65.Re, 87.64.-t, 87.64.kv, 87.64.mn

\section{Introduction}

Multiphoton endoscopy (MPE) is a powerful tool for noninvasive and high resolution imaging of biological tissues. MPE applies nonlinear optical effects such as two-photon fluorescence (TPF) and second harmonic generation (SHG). To excite TPF and SHG signals femtosecond pulses are required. Therefore, the first major issue in MPE is how to efficiently deliver single-mode femtosecond excitation signal and collect multimode two-photon fluorescence response.

There are known two major designs of endoscopic probe [1-5] and [6-11]. The first configuration is shown in Fig. 1. In this approach two-fiber are used. A single-mode fiber (SMF) is used to deliver and focus one-mode femtosecond signals and the multimode fiber with large core diameter and high numerical apeture (NA) to collect TPF signals.

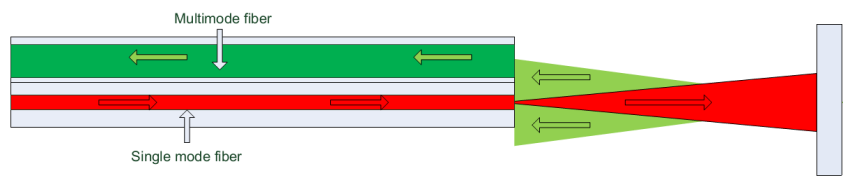

Fig. 1. Schematic of fiber-optic two-photon fluorescence endoscope in two-fiber configuration.

The SMF cannot be used to collect TPF signals due to small core diameter which causes low collection efficiency. Another problem with the applications is that the excitation area does not cover exactly the collecting area. To solve this problem researchers utilize microelectromechanical systems as shown in Fig. 2.

Such approach is generally sensitive to misalignment and has suboptimal throughput. Additionally such solution is more complicated than the second configuration

\footnotetext{
* corresponding author; e-mail: Hanna.Stawska@pwr.wroc.pl
}

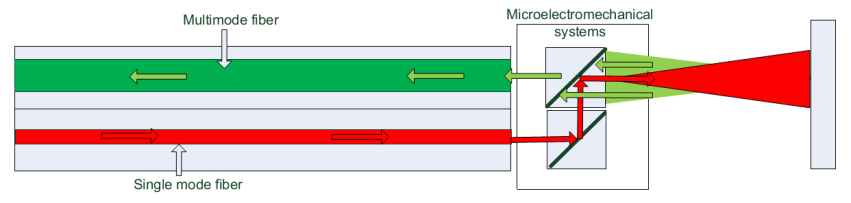

Fig. 2. Schematic of fiber-optic two-photon fluorescence endoscope in two-fiber configuration with microelectromechanical systems [1].

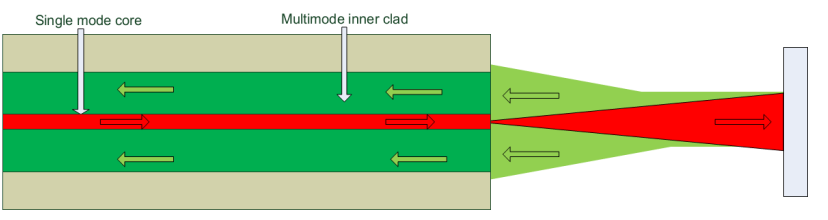

Fig. 3. Schematic of fiber-optic two-photon fluorescence endoscope in configuration with DC PCF.

which utilizes double-cladding photonic crystal fibers DC PCF as shown in Fig. 3. In this case femtosecond pulses are delivered by the single-mode core and TPF signals are collected by both the core and the multimode inner cladding of DC PCF and this increases the collection efficiency. The large core size of the DC PCF can also minimize nonlinear effect inside the fiber.

Typical length of fiber applied in endoscopy is about $2 \mathrm{~m}$. Therefore, for such short length one can assume that signal attenuation could be neglected. In the presented area we must consider chromatic dispersion which causes femtosecond pulses to spread to picoseconds pulses when propagating in optical fibers, thereby deteriorating the excitation efficiency of TPF signals. Tang et al. [5] have shown that dispersion of DC PCF broadens the $170 \mathrm{fs}$ pulse to $2.5 \mathrm{ps}$. Therefore the management of dispersion is a critical issue in the design of TPF endoscopy.

One way to realize this is to use dispersion compensating systems. For example to precompensate the disper- 
sion and to compress the pulse width back to the femtosecond regime at the end of the fiber, Tang et al. have proposed to insert a grating pair before the fiber and $\mathrm{Wu}$ et al. [3] have used bandgap fiber negatively prechirping the pulse. But these compensating systems have the same limitations. Due to utilization of bulb optics they are sensitive to misalignments and have limited excitation throughput. In general in mentioning systems there are used.

The second way is to design DC PCF with small dispersion. In this paper a new design of double cladding small dispersion photonic crystal fiber is proposed.

\section{Design principle}

To design a fiber which combines advantages of PBG fibers featuring positive dispersion (to compensate the negative dispersion of DCF) and DCF with relatively good collecting efficiency we have checked the idea of construction of photonic, double cladding bandgap fibers in low-index-contrast material systems and in high-index-contrast material systems. We consider two fiber constructions: first, with germanium doped silica rods and second, with hollow core.

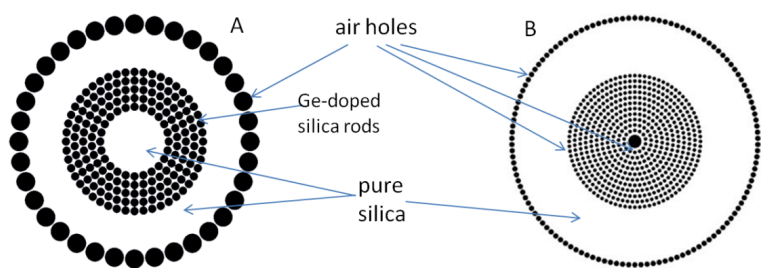

Fig. 4. Cross-section of circular lattice DC PCF with germanium doped silica rods (A) and with hollow core $(\mathrm{B})$.

Figure $4 \mathrm{~A}$ shows the cross-section of proposed fibers with germanium doped silica rods and Fig. 4B - with hollow core and air holes. Differently from other designs, in our approach a circular lattice is used. To define the structure of this DC PCF, seven parameters, inner cladding hole to hole radial distance $\Lambda_{1}$, inner cladding air-hole diameter $d_{1}$, outer cladding hole to hole radial distance $\Lambda_{2}$, inner cladding cavity size of missing concentric circles $N_{2 \max }$, inner cladding number of concentric circles $N_{1 \text { max }}$, outer cladding air-hole diameter $d_{2}$, outer cladding cavity size of missing concentric circles $N_{2}$ min, outer cladding number of concentric circles $N_{2} \max$ and radius of the core $R$, are used.

To analyze our PCFs we used the commercially available software package Lumerical Mode Solution which relies on a fully-vectorial and rigorous finite difference method for solving the wave equation. Calculation output from this software is field distribution, its effective index and losses.

\section{Numerical results}

Our goal was to achieve a fiber with low dispersion. In order to accomplish this target there was performed analysis of relation between dispersion and geometrical structure of examined fibers.

During calculations we obtained effective index of the fundamental mode. In this way it was possible carrying out the following analysis.

The effective refractive index of the fundamental mode is given by $n_{\text {eff }}=\beta / k_{0}$ where $\beta$ is propagation constant and $k_{0}$ is the free-space wave number. The group velocity dispersion $D$ can be calculated from the modal effective index [5]:

$$
D(\lambda)=-\left(\frac{\lambda}{c}\right) \frac{\mathrm{d}^{2} n_{\mathrm{eff}}}{\mathrm{d} \lambda^{2}},
$$

where $c$ is velocity of the light. On the other hand, the total dispersion is calculated as the sum of the geometrical dispersion (waveguide dispersion) and the material dispersion in the first order approximation [6]:

$$
D(\lambda)=D_{\mathrm{g}}(\lambda)+\Gamma(\lambda) D_{\mathrm{m}}(\lambda),
$$

where $\Gamma$ is the confinement factor in silica, which is close to unity for the most practical PCF's as the almost all energy concentrates in the silica with high refractive index. The material dispersion $D_{\mathrm{m}}$ can be obtained directly from the general three term Sellmeier formula [6]:

$$
n^{2}(\lambda)=1+\sum_{i=1}^{3} \frac{B_{i} \lambda^{2}}{\lambda^{2}-\lambda_{i}^{2}}
$$

In order to calculate material dispersion coefficients presented in Table I were applied for pure silica and germanium doped silica.

Sellmeier coefficient for pure silica and Ge-doped silica.

TABLE I

\begin{tabular}{c|c|c|c|c|c|c}
\hline \hline Sellmeier's coefficients & B1 & B2 & B3 & $\lambda_{1}[\mu \mathrm{m}]$ & $\lambda_{2}[\mu \mathrm{m}]$ & $\lambda_{3}[\mu \mathrm{m}]$ \\
\hline pure silica & 0.6961663 & 0.4079426 & 0.897479 & 0.0684043 & 0.1162414 & 9.896161 \\
Ge-doped $3.5 \mathrm{~m} / \mathrm{o} \mathrm{GeO} \mathrm{GeO}_{2}, 96.5 \mathrm{~m} / \mathrm{o} \mathrm{SiO}_{2}$ & 0.7042038 & 0.4160032 & 0.9074049 & 0.0514415 & 0.1291600 & 9.896156
\end{tabular}

During our experiments we have simulated a lot of configuration of fiber parameters such as hole to hole/rods to rods distances, number of rings, number of holes/rods in the particular rings. Conclusion is that waveguide dispersion is strongly related to the design parameters of the PCFs and therefore can be optimized to achieve de- 
sired dispersion properties. To find the optimal solution it is required to check a lot of parameter configuration. For the time being we found two best but not optimal solutions. The first one was Ge-doped fiber. In Fig. 5 near field electric field intensities are presented for fundamental mode at $800 \mathrm{~nm}$ (A) and for sample higher order mode at $500 \mathrm{~nm}$ (B). The solution was obtained for the geometrical parameters presented in Table II.

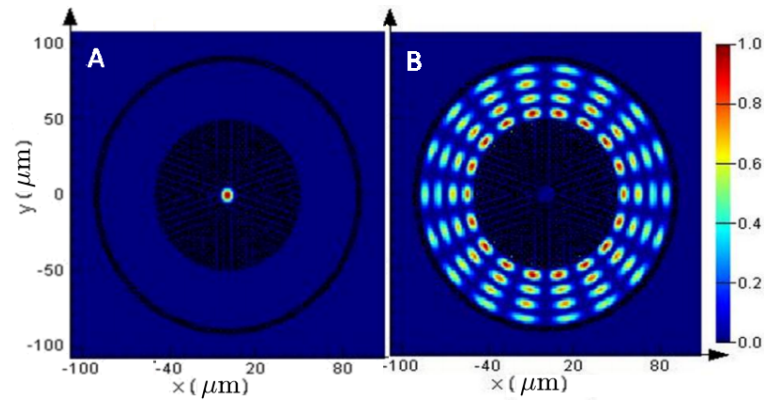

Fig. 5. Near-field electric field intensities for Ge-doped fiber. (A) The fundamental mode at $800 \mathrm{~nm}$, (B) sample higher-order mode at $500 \mathrm{~nm}$.

In Fig. 5A the excitation signal is presented and the whole energy is concentrated in the core. In Fig. 5B the sample simulated response from the tissue is shown and energy is concentrated in the clad.

Geometrical parameters of Ge-doped fiber.

TABLE II

\begin{tabular}{c|c||c|c||c|c}
\hline \hline$\Lambda_{1}$ & $4 \mu \mathrm{m}$ & $\Lambda_{2}$ & $5 \mu \mathrm{m}$ & core & $13 \mu \mathrm{m}$ \\
$d_{1}$ & $3 \mu \mathrm{m}$ & $d_{2}$ & $4 \mu \mathrm{m}$ & inner clad & $90 \mu \mathrm{m}$ \\
$N_{1 \min }$ & 2 & $N_{2 \min }$ & 18 & outer clad & $180 \mu \mathrm{m}$ \\
$N_{1 \max }$ & 12 & $N_{2 \max }$ & 18 & $A_{\text {eff }}$ & $98 \mu \mathrm{m}$
\end{tabular}

As we can see in Fig. 6A dispersion of Ge-doped fiber is strongly nonlinear and little change of wavelength makes big change of dispersion. This solution is not practical because it is troublesome to tune the laser so exactly. Obtained losses are relatively low, in range $10^{-9}$ as shown in Fig. 6B. At $800 \mathrm{~nm}$ dispersion is equal to $-2 \mathrm{ps} / \mathrm{nm} \mathrm{km}$.

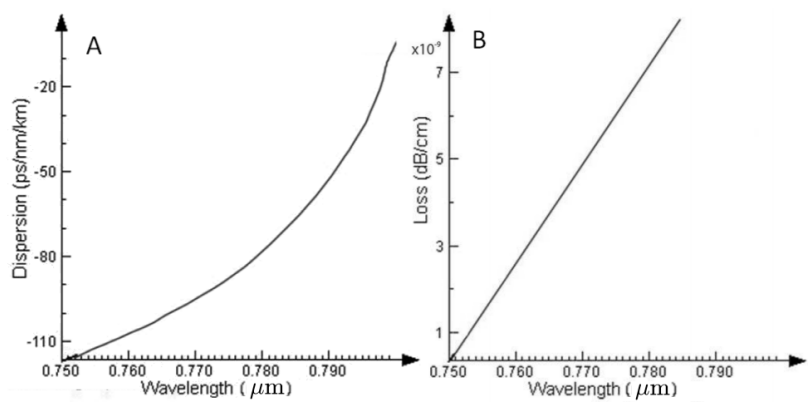

Fig. 6. Dispersion (A) and losses (B) Ge-doped fiber.

In Fig. 7 near field electric field intensities are presented for hollow core fiber. In this case the characteristic of near field electric field intensities are similar to

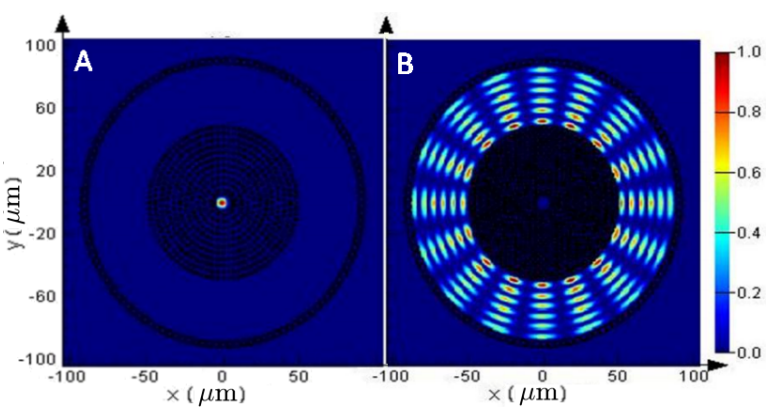

Fig. 7. Near-field electric field intensities for hollow core fiber. (A) The fundamental mode at $800 \mathrm{~nm}$, (B) sample higher-order mode at $500 \mathrm{~nm}$.

Ge-doped but dispersion (Fig. 8A) is different, is more linear and the slope of characteristic is lower. Losses (Fig. 8B) are also very low.

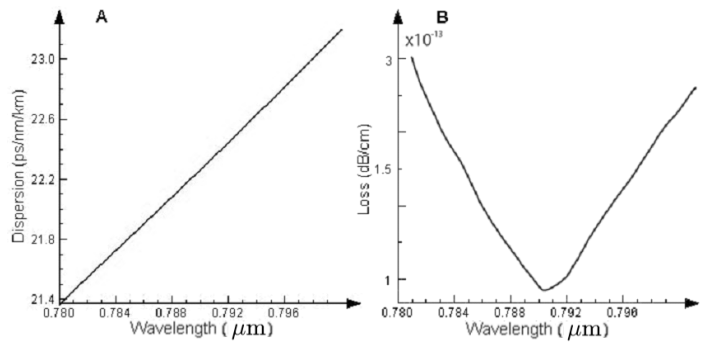

Fig. 8. Dispersion (A) and losses (B) hollow core fiber.

In Table III there are presented geometrical parameters of hollow core fiber. For the hollow core fiber and Ge-doped fiber with the same geometrical parameter, the effective mode area $A_{\text {eff }}$ is bigger for the Ge-doped fiber.

Application of ultrafast fibers is related with high impulse power which could lead to material damage. That way it is important to design solid core fiber with large effective mode area.

Geometrical parameters of hollow core fiber.

TABLE III

\begin{tabular}{c|c||c|c||c|c}
\hline \hline$\Lambda_{1}$ & $4 \mu \mathrm{m}$ & $\Lambda_{2}$ & $5 \mu \mathrm{m}$ & core & $11 \mu \mathrm{m}$ \\
$d_{1}$ & $3 \mu \mathrm{m}$ & $d_{2}$ & $4 \mu \mathrm{m}$ & inner clad & $90 \mu \mathrm{m}$ \\
$N_{1 \mathrm{~min}}$ & 2 & $N_{2 \min }$ & 18 & outer clad & $180 \mu \mathrm{m}$ \\
$N_{1 \max }$ & 12 & $N_{2 \max }$ & 18 & $A_{\text {eff }}$ & $38 \mu \mathrm{m}$
\end{tabular}

\section{Calculations of broadening factor}

The propagation of pulses through a fiber is usually described by two parameters called the non-linear length $L_{\mathrm{d}}$ and the dispersion length $L_{\mathrm{n}}$

$$
\begin{aligned}
L_{\mathrm{n}} & =-\frac{A_{\mathrm{eff}} \lambda}{2 \pi n_{2} P}, \\
L_{\mathrm{d}} & =-\frac{2 \pi c}{\lambda^{2}} \frac{T_{0}}{D} .
\end{aligned}
$$

If the pulses become shorter than $\left(T_{0}<50 \mathrm{ps}\right)$ and the 
dispersion length becomes comparable to the fiber length, it turns out to be necessary to consider the combined effects of GVD and SPM. In this case when an unchirped Gaussian pulse is incident at the input end of a fiber of length $L$, the broadening factor is given by [6]:

$$
\begin{aligned}
f & =\frac{\sigma}{\sigma}_{0}=\left[1+\sqrt{2} \phi_{\max } \frac{L}{L_{\mathrm{D}}}\right. \\
& \left.+\left(1+\frac{4}{3 \sqrt{3}} \phi_{\max }^{2}\right) \frac{L^{2}}{L_{\mathrm{d}}^{2}}\right]^{1 / 2},
\end{aligned}
$$

where $\phi_{\max }$ is the SPM-induced maximum phase shift given in

$$
\phi_{\max }=\gamma P_{0} L_{\text {eff }},
$$

where

$$
L_{\mathrm{eff}}=\frac{1-\mathrm{e}^{-\alpha L}}{\alpha},
$$

and $\alpha$ - fiber losses. Calculations of broadening factor for proposed fibers are presented in Table IV. The result is obtained with assumption that fiber length is $2 \mathrm{~m}$ and initial pulse width is $100 \mathrm{fs}$.

Calculation of broadening factor.

TABLE IV

\begin{tabular}{c|c|c|c|c|c|c}
\hline \hline & $\begin{array}{c}\text { Dispersion }(D) \text { at } 800 \mathrm{~nm} \\
{[\mathrm{ps} /(\mathrm{nm} \mathrm{km})]}\end{array}$ & $\begin{array}{c}\text { Nonlinear refractive } \\
\text { index }\left(n_{2}\right)\left[\mathrm{m}^{2} / \mathrm{W}\right]\end{array}$ & $L[\mathrm{~m}]$ & $L_{\mathrm{d}}[\mathrm{m}]$ & $L_{\mathrm{n}}[\mathrm{m}]$ & $\begin{array}{c}\text { Broadening } \\
\text { factor } f\end{array}$ \\
\hline hollow core DC PCF & 23 & $3.0 \times 10^{-23}$ & 2 & 1.54 & $8.0639 \times 10^{6}$ & 1.69 \\
Ge-doped DC PCF & -2 & $3.2 \times 10^{-20}$ & 2 & 14.73 & $2.0796 \times 10^{4}$ & 1.01
\end{tabular}

Results obtained in Table IV show that $2 \mathrm{~m}$ Ge-doped fiber with dispersion equal to -2 does not make impulse broader - factor $f$ is 1.01. Although the dispersion characteristic for presented hollow core DC PCF is linear and has lower slope but value of dispersion at $800 \mathrm{~nm}$ is still high and consequently the broadening factor is meaningful. Due to this fact there is required further fiber work out.

\section{Conclusion}

First investigation showed up that solution is feasible. It means that it is possible to design double cladding small dispersion photonic crystal fiber to guide ultrashort pulse at $800 \mathrm{~nm}$. Application of these fibers significantly simplifies construction of nonlinear imaging systems by elimination of dispersion compensation systems. Additionally, hollow core fibers can be used to stretch pulse width, resulting in higher efficiency of autofluorescence.

\section{Acknowledgments}

Calculations have been carried out in Wrocław Centre for Networking and Supercomputing (http://www.wcss.wroc.pl), Grant No. 184.

\section{References}

[1] Advances in Lasers and Electro Optics, Eds. N. Costa, A. Cartaxo, Artech House, London 2010.

[2] Y. Jing Young, Multiphoton Microscopy in the Biomedical Sciences, Plenum Press, New York 2005.

[3] Y. Wu, Y. Leng, X. Li, D.J. Mac Donald, M.J. Cobb, J. Biomed. Opt. 14, 024066 (2009).

[4] J. Ignac-Nowicka, T. Pustelny, Z. Opilski, W. Maciak, W. Jakubik, M. Urbanczyk, Opt. Eng. 42, 2978 (2003).

[5] S. Tang, S, W. Jung, D. McCormick, T. Xie, J. Su, Y.C. Ahn, B.J. Tromberg, Z. Chen, J. Biomed. Opt. 14, 0398223 (2009).

[6] P. Govind, G. Agrawal, Nonlinear Fiber Optics, Academic Press, London 2007.

[7] E. Bereś-Pawlik, F. Dybała, W. Michalski, D. Dus, Opt. Appl. 34, 87 (2004).

[8] E. Bereś-Pawlik, K. Gąsiorek, Z. Kulas, M. Rząca, Acta Phys. Pol. A 116, 254 (2009).

[9] T. Pustelny, M. Grabka, Acta Phys. Pol. A 116, 385 (2009).

[10] Z. Kulas, E. Bereś-Pawlik, T. Kręcicki, J. Non-Cryst. Solids 352, 2468 (2006).

[11] T. Pustelny, J. Ignac-Nowicka, B. Jarzabek, A. Burian, Opt. Appl. 34, 551 (2004). 